\title{
Serviços de Atendimento Móvel de Urgência (Samu): uma revisão integrativa
}

\author{
Mobile Emergency Care Services (Mecs): an integrative review \\ Servicios Móviles de Atención de Emergencia (Smae): una revisión integradora
}

Tais de Oliveira Marques

ORCID: https://orcid.org/0000-0002-3885-0137

Universidade Federal de Juiz de Fora, Brasil

E-mail: tais.marques@hotmail.com

Laércio Deleon de Melo

ORCID: https://orcid.org/0000-0002-8470-7040

Universidade do Estado do Rio de Janeiro, Brasil

E-mail: laerciod128@hotmail.com

Felipe Eduardo Taroco

ORCID: https://orcid.org/0000-0002-8530-5780

Centro Universitário Estácio de Juiz de Fora, Brasil

E-mail: fisiofelipe91@gmail.com

Rafaela Maria de Lima Duarte

ORCID: https://orcid.org/0000-0001-6802-9772

Universidade Salgado de Oliveira, Juiz de Fora, Brasil

E-mail: rhafinha_@hotmail.com

Huyara Dias de Lima

ORCID: https://orcid.org/0000-0002-6089-5518

Faculdade de Ciências Médicas e da Saúde de Juiz de Fora, Brasil

E-mail: huyaradias@gmail.com

\begin{abstract}
Resumo
Objetivou-se elencar quais são os temas que estão sendo publicados sobre os serviços de atendimento nas unidades móveis de urgência. Foram atendidas as seis etapas da revisão integrativa, visando responder à questão de pesquisa: quais são as temáticas que têm sido publicadas nos últimos dez anos sobre o atendimento nas unidades móveis de urgência? Os dados foram coletados em 2016, com delimitação temporal de dez anos, artigos no idioma português, que estivessem indexados na íntegra na Biblioteca Virtual de Saúde, a partir dos descritores: "emergências"; "ambulâncias"; "socorro de urgência". Foram selecionadas 14 publicações, e os estudos mais recentes datavam de 2012 e abordavam temas relacionados à gestão dos serviços de atendimento móvel de saúde. Conclui-se que ainda há necessidade de realização de novas investigações que busquem propor estratégias para organizar e melhor divulgar o trabalho desse serviço, assim como a realização de pesquisas que reforcem o compromisso da atuação em rede com os outros níveis de atenção à saúde, dedicando esforços para se colocar em prática o estabelecido na Política Nacional de Atenção às Urgências.
\end{abstract}

Palavras-chave: Ambulâncias; Emergências; Socorro de urgência.

\begin{abstract}
The objective was to list what are the topics that are being published about care services in mobile emergency units. The six stages of the integrative review were attended to, aiming to answer the research question: what are the themes that have been published in the last ten years about care in mobile emergency units? Data were collected in 2016, with a ten-year time limit, articles in Portuguese, that were fully indexed in the Virtual Health Library, using the descriptors: "emergencies"; "Ambulances"; "Emergency relief". 14 publications were selected, and the most recent studies dated 2012 and addressed topics related to the management of mobile health care services. It is concluded that there is still a need to carry out new investigations that seek to propose strategies to organize and better publicize the work of this service, as well as conducting research that reinforces the commitment of working in networks with other levels of health care, dedicating efforts to put into practice what is established in the National Emergency Care Policy. Keywords: Ambulances; Emergencies; Emergency relief.
\end{abstract}

\section{Resumen}

El objetivo fue enumerar cuáles son los temas que se están publicando sobre los servicios de atención en las unidades móviles de emergencia. Se atendieron las seis etapas de la revisión integradora, con el objetivo de dar respuesta a la pregunta de investigación: cuáles son los temas que se han publicado en los últimos diez años sobre la atención en unidades móviles de emergencia? Los datos fueron recolectados en 2016, con un límite de tiempo de diez años, artículos en portugués, que fueron completamente indexados en la Biblioteca Virtual en Salud, utilizando los 
descriptores: "emergencias"; "Ambulancias"; "Ayuda de emergencia". Se seleccionaron 14 publicaciones y los estudios más recientes datan de 2012 y abordan temas relacionados con la gestión de los servicios móviles de salud. Se concluye que aún existe la necesidad de realizar nuevas investigaciones que busquen proponer estrategias para organizar y dar a conocer mejor el trabajo de este servicio, así como realizar investigaciones que refuercen el compromiso de trabajar en red con otros niveles de atención de la salud, dedicando esfuerzos para poner en práctica lo establecido en la Política Nacional de Atención de Emergencias.

Palabras clave: Ambulancias; Urgencias médicas; Socorro de urgencia.

\section{Introdução}

O Atendimento Pré-hospitalar (APH) surgiu em 1893 no Brasil, na cidade do Rio de Janeiro, interligado ao Setor de Saúde e Segurança Pública. O mesmo se constitui como uma estratégia de intervenção por parte do Estado, para ofertar atendimento precoce e ágil, com transporte adequado a um serviço emergencial, visando assim reduzir riscos, complicações e aumentar a sobrevida das vítimas (Silva, Mariot, \& Riegel, 2020).

No contexto do Sistema Único de Saúde (SUS), o APH divide-se em dois tipos de atendimento: pré-hospitalar fixo e móvel, sendo que o foco deste estudo é o Serviço de Atendimento Móvel de Urgência (Samu), cuja finalidade é prestar atendimento emergencial móvel e ações de salvamento e resgate, com qualidade, em qualquer lugar (residências, locais de trabalho e vias públicas) (Silva et al., 2009; Gomes, \& Miranda, 2020).

O Samu é requisitado de forma gratuita pelo telefone 192 e atende ininterruptamente durante 24 horas, todos os dias. O telefonema do solicitante é avaliado por técnicos da central de regulação e posteriormente pelo médico regulador, que, com base em escuta e entendimento da situação, faz o diagnóstico e a classificação da urgência. Posteriormente esse profissional tece orientações necessárias para o atendimento à vítima. Considerando a gravidade e a urgência da situação, ele já aciona o envio de Unidade de Suporte Básico (USB) ou o Avançado de Vida (SAV). A primeira é tripulada por técnico de enfermagem e condutor do veículo e a segunda, por médico, enfermeiro e condutor do veículo (Rosa et al., 2020; Silva, Mariot, \& Riegel, 2020).

$\mathrm{O}$ atendimento via telefone é denominado telemedicina, sendo o mesmo norteado por um protocolo que auxilia o profissional na tomada de decisões. Esse serviço conta com o aporte de uma equipe multiprofissional formada por médicos, enfermeiros, técnicos de enfermagem, condutores e Telefonistas Auxiliares de Regulação Médica (Tarms). Esse serviço possibilita organizar o acesso aos atendimentos de emergência, ao leito hospitalar e à rede de forma mais ampla, favorecendo o atendimento integral às urgências em todos os níveis de atenção do sistema (O’Dwyer \& Mattos, 2012; Zerbetto Filho, de Souza \& Carvalho, 2020).

Desse modo, o objetivo de tal serviço é atender e/ou transportar a vítima para o serviço de saúde, posteriormente à ocorrência de uma determinada situação ou agravo à sua saúde, de natureza clínica, cirúrgica, obstétrica, traumática ou psiquiátrica, tendo em vista a redução de complicações, sequelas ou os riscos de morte (Brasil, 2006).

Segundo Veronese, Oliveira e Nast (2012), parada cardiorrespiratória, dificuldade respiratória severa, convulsões, lesões por acidentes de trânsito e quedas, queimaduras, afogamentos, agressões, choques elétricos, bem como outros agravos são situações que devem ser atendidas pelo Samu, uma vez que envolvem risco de vida iminente quando ocorridas no ambiente extra-hospitalar.

A Política Nacional de Atenção às Urgências (Pnau), instituída em 2003, estabelece normas para a organização dos serviços de APH públicos e privados nas esferas estadual, regional e municipal, bem como define os componentes desse serviço (Brasil, 2003). Logo, o Samu é uma estratégia de alta complexidade e indispensável ao funcionamento do serviço de saúde do Brasil, que busca fortalecer a implantação das diretrizes preconizadas pela Pnau. Assim, de acordo com os princípios do SUS, tal serviço combina ações de prevenção, atenção individual e coletiva. Destarte, cabe mencionar que o Samu foi o primeiro órgão componente da Pnau a ser implantado no Brasil (O’Dwyer \& Mattos, 2012). 
Silva et al. (2009) destacam que os desafios na instituição do Samu são diversos. No entanto, em detrimento de todas as dificuldades gerenciais, organizacionais e operacionais, há que se reconhecer e ressaltar a importância e a necessidade dos serviços para a sociedade, uma vez que a implementação deste, por meio do sistema de regulação, padronização e especificação do atendimento, tornou-se uma realidade a salvar vidas com eficácia.

Diante do exposto, emergiu a seguinte questão norteadora: quais são as temáticas que têm sido publicadas nos últimos dez anos sobre o Samu? Justifica-se a importância deste estudo diante da possibilidade de ampliar o conhecimento e a divulgação de novos conhecimentos sobre o Samu no contexto da assistência multiprofissional. Objetivou-se, portanto, elencar quais são os temas que estão sendo publicados sobre os serviços de atendimento nas unidades móveis de urgência.

\section{Metodologia}

O delineamento de investigação adotado foi o de uma revisão integrativa da literatura, no que se refere aos temas abordados na literatura científica sobre os serviços de atendimento móvel de urgência. A revisão integrativa é, atualmente, o método mais utilizado na prática baseada em evidências, uma vez que estimula a utilização dos resultados de pesquisas na assistência à saúde, além de possibilitar a implementação das evidências na prática clínica (Sousa, Marques-Vieira, Severino \& Antunes, 2017).

Cabe mencionar que foram atendidas de forma criteriosa as seis etapas metodológicas da revisão integrativa, a saber: na primeira, foi feita a eleição do tema que precede a elaboração da questão norteadora; na segunda, definida pela seleção dos critérios de inclusão e exclusão, teve início a busca exaustiva nas bases de dados, para triagem dos artigos; na terceira, foram organizadas as informações encontradas nos artigos; na quarta, foi realizada a avaliação dos artigos de modo crítico e observador, quando ocorreu a seleção ou o descarte de artigo segundo os objetivos do estudo. Já na quinta etapa, os artigos selecionados foram interpretados pelo pesquisador e, com base na leitura teórica do tema em questão, foi feita a sua classificação segundo o nível de evidência. Nesse momento, é possível realizar propostas para futuras pesquisas e/ou refutar resultados. Na sexta e última etapa, fez-se a apresentação da revisão propriamente dita, que corresponde à elaboração do documento descritivo das etapas realizadas e dos resultados encontrados (Souza et al., 2017; Melo et al., 2020).

A primeira etapa teve início a partir da seguinte pergunta norteadora da pesquisa: quais são as temáticas que têm sido publicadas (inter)nacionalmente nos últimos dez anos sobre atendimento nas unidades móveis de urgência? Considerando a referida questão, foram selecionados nos Descritores em Ciências da Saúde (DeCS), segundo a terminologia em saúde na Biblioteca Virtual de Saúde (BVS) os descritores: "Emergências", "Ambulâncias" e "Socorro de urgência" e seus respectivos correspondentes em inglês e espanhol, sendo utilizado no cruzamento dos descritores o operador booleano AND.

$\mathrm{Na}$ segunda etapa, com os descritores escolhidos, iniciou-se a seleção dos artigos divulgados na BVS até 2016. Nessa fase, utilizaram-se como critérios de inclusão: publicações (inter)nacionais, com textos completos disponíveis, e estudos que tivessem sido publicados no período temporal de 2006 até 2016. Finalmente, foram excluídos os artigos que não apresentaram relação com a temática, os repetidos e as monografias.

\section{Resultados e Discussão}

Foram pré-selecionados 14 artigos e, após o final da triagem dos estudos baseados nos critérios de inclusão e exclusão, foi selecionado o total de cinco publicações científicas que abordaram o tema investigado. Cabe mencionar que, do total de artigos, foram excluídos dois por não compreenderem a temática pesquisada, quatro por estarem repetidos, dois por se tratarem de monografias e outros dois por não estarem disponíveis na íntegra para leitura.

Assim a amostra final se compôs de cinco artigos, os quais foram analisados por meio da leitura na íntegra, sendo aplicada a descrição das seguintes variáveis selecionadas para análise no estudo: ano de publicação, local de realização da 
pesquisa, área do periódico e metodologia do estudo (Tabela 1), título do artigo e o tema abordado sobre o serviço de atendimento móvel (Quadro 1).

Tabela 1: Caracterização dos artigos selecionados para análise dos dados, segundo ano de publicação, local do estudo, área do conhecimento e metodologia, Juiz de Fora, MG, Brasil, 2021.

\begin{tabular}{ll}
\hline Variável & Número de artigos \\
\hline Ano de publicação & 1 \\
2007 & 1 \\
2009 & 3 \\
2012 & 5 \\
Total & \\
Local da pesquisa & 1 \\
Sudeste & 2 \\
Sul & 2 \\
Nordeste & 5 \\
Total & \\
Área do Periódico & 3 \\
Enfermagem & 2 \\
Saúde pública & 5 \\
Total & \\
Metodologia & 2 \\
Pesquisa qualitativa & 1 \\
Pesquisa quantitativa & 1 \\
Pesquisa qualitativa-quantitativa & 1 \\
Estudo transversal & 5 \\
Total & \\
\hline
\end{tabular}

Fonte: Autores.

Todos os artigos são de autoria de enfermeiros e publicados em periódicos da área. Três (60\%) dos trabalhos encontrados foram publicados no ano de 2012, um (20\%) em 2009 e outro (20\%) em 2007. Dois estudos (40\%) foram realizados na região Sul do Brasil, outros dois foram realizados na região Nordeste e somente um (20\%) na região Sudeste. Três $(60 \%)$ estudos foram publicados em revistas de Enfermagem, e outros dois (40\%) em revista de saúde pública. A metodologia dos estudos encontrados se mostrou variada, sendo que, em dois estudos (40\%), foi utilizada a metodologia qualitativa. Esses dados se referem à caracterização dos estudos encontrados conforme apresentado na Tabela 1.

Ressalta-se que não foi encontrada nenhuma publicação datada do ano de 2016, bem como foi observado que há poucos estudos nacionais que versam sobre o serviço de atendimento móvel especificamente. Os temas encontrados foram sintetizados, divididos e apresentados no Quadro 1 para melhor compreensão e visualização. 
Quadro 1: Número, títulos dos artigos e descrição dos temas sobre serviço de atendimento móvel, Juiz de Fora, MG, Brasil, 2021.

\begin{tabular}{|c|c|}
\hline Número e Título do artigo & Temas sobre serviço de atendimento móvel \\
\hline $\begin{array}{l}\text { 1. Serviço de atendimento móvel de } \\
\text { urgência (Samu) no município de Juazeiro } \\
\text { (BA): principais especialidades } \\
\text { demandadas }\end{array}$ & $\begin{array}{l}\text { a) As principais especialidades demandadas pelo Samu do município de Juazeiro (BA), } \\
\text { como clínica médica, seguida da ortopedia e traumatologia e também a geriatria. } \\
\text { b) Perfil das demandas de atendimento. } \\
\text { b.1) Demanda maior de mulheres é para clínica médica, obstetrícia e ginecologia e geriatria. } \\
\text { b.2) Demandas de homens: ortopedia e traumatologia clínica médica e geriatria. } \\
\text { b.3) Faixa etária mais frequente que procura os serviços de urgência no município está entre } \\
21 \text { e } 30 \text { anos, com um percentual eminentemente jovem. } \\
\text { c) Necessidade de projetos de educação em saúde para orientar a população sobre os reais } \\
\text { objetivos no atendimento do SAM. } \\
\text { d) Recomendação de realização de estudos e pesquisas que envolvam o serviço de } \\
\text { atendimento móvel nas localidades onde atuam. }\end{array}$ \\
\hline $\begin{array}{l}\text { 2. Caracterização da demanda não } \\
\text { pertinente ao Samu de Porto Alegre: estudo } \\
\text { descritivo }\end{array}$ & $\begin{array}{l}\text { a) Apresentar a caracterização de demandas não pertinentes (DNP) por Gerências (GER) da } \\
\text { Secretaria Municipal de Saúde. b) Detalhamento das características das DNPs. } \\
\text { b.1) Dia da semana em que há maior ocorrência. } \\
\text { b.2) Faixa horária da demanda não pertinente. } \\
\text { b.3) O subtipo de socorro. } \\
\text { c) Recomenda: trabalho contínuo, que promova a competência da população para agir com } \\
\text { autonomia diante de um problema urgente de saúde. } \\
\text { c.1) Ações para conceder orientações para população sobre o funcionamento do Samu. } \\
\text { c.2) Banco de dados do Samu seja preenchido de modo completo e correto pelas telefonistas } \\
\text { auxiliares de regulação médica (Tarms) e pelos médicos regulares (MR), a fim de que as } \\
\text { pesquisas possam dispor de informações mais precisas sobre as características da demanda } \\
\text { ao serviço. }\end{array}$ \\
\hline $\begin{array}{l}\text { 3. Atendimento pré-hospitalar móvel em } \\
\text { Fortaleza, Ceará: a visão dos profissionais } \\
\text { envolvidos }\end{array}$ & $\begin{array}{l}\text { a) Ponto de vista dos profissionais que atuam no pré-hospitalar sobre várias questões que } \\
\text { envolvem o funcionamento do serviço. } \\
\text { a.1) Os profissionais }(60,7 \%) \text { relataram que a estrutura física do Samu deixa a desejar. } \\
\text { a.2) Escassez de recursos materiais foi apontada por } 82,0 \% \text { dos profissionais. } \\
\text { a.3) Falhas na integração com outros serviços. } \\
\text { a.4) Necessidade de capacitação/ educação permanente para o profissional do Samu. } \\
\text { a.5) Garantia de acesso da equipe do Samu e o conhecimento sobre a Política Nacional de } \\
\text { Atenção às Urgências. } \\
\text { b) Garantia de implantação do Samu e a promoção de seu aperfeiçoamento e constante } \\
\text { avaliação. }\end{array}$ \\
\hline $\begin{array}{l}\text { 4. Acidentes de trabalho com motoristas de } \\
\text { Ambulâncias que realizam socorro de }\end{array}$ & $\begin{array}{l}\text { a) Descreve questões que implicam e favorecem a ocorrência de Acidente de Trabalho, com } \\
\text { os motoristas de ambulâncias. }\end{array}$ \\
\hline
\end{tabular}




\begin{tabular}{|c|c|}
\hline urgência & $\begin{array}{l}\text { a.1) Ausência de capacitação para os condutores. } \\
\text { a.2) Déficit salarial, pois muitos trabalhadores possuem outro trabalho. } \\
\text { a.3) Falta de manutenção preventiva dos veículos. } \\
\text { a.4) Execução de tarefas que não são de sua competência. } \\
\text { a.5 ) Desgaste físico. }\end{array}$ \\
\hline $\begin{array}{l}\text { 5. Risco de vida e natureza do Samu: } \\
\text { demanda não pertinente e implicações para } \\
\text { a enfermagem }\end{array}$ & $\begin{array}{l}\text { a) Aponta questões que fazem menção à gestão dos serviços no âmbito do SUS e, em } \\
\text { particular, à gestão do Samu e à Enfermagem. } \\
\text { b) Discute sobre gestão do Samu, visando colaborar para o planejamento de ações de } \\
\text { atenção às urgências. } \\
\text { c) Discute sobre o papel da Enfermagem no que tange a sua atuação nas atividades de } \\
\text { gerência deste serviço. } \\
\text { d) Aponta que a subjetividade na avaliação do risco de vida causa problemas entre } \\
\text { profissionais e usuários. Sugere necessidade de orientação para população sobre o serviço. } \\
\text { e) Identifica que os usuários, muitas vezes, reconhecem o serviço do Samu como } \\
\text { possibilidade para ter acesso a atendimento médico rápido e acesso a medicamentos. } \\
\text { f) Identifica que usuários possuem conhecimento, porém a percepção sobre o Samu é } \\
\text { equivocada. } \\
\text { g) Atuação da enfermagem como potencial estratégia para redução das DNPs. }\end{array}$ \\
\hline
\end{tabular}

Fonte: Autores.

As temáticas discutidas nos trabalhos sobre o Samu são diversas, porém se observou um maior aprofundamento dos conteúdos relativos a gestão e funcionamento de tais serviços, a discussão sobre as questões pontuadas pelas pesquisas acima citadas está disposta em dois núcleos temáticos - Gestão dos Serviços de Atendimento Móvel: funcionamento; Gestão dos Serviços de Atendimento Móvel: Capacitação profissional e Ações educativas para população.

\subsection{Gestão dos Serviços de Atendimento Móvel: funcionamento}

O artigo de autoria de Rocha, Morais e Benevides (2012) aborda temas relativos às especialidades cujas demandas possuem maior procura nos serviços de Urgência e Emergência, além de traçar um perfil dos usuários segundo tais demandas. Os resultados do referido estudo revelam que a maioria das demandas dos atendimentos foram solicitadas por mulheres e que, em geral, tal solicitação é feita por um público jovem. Tal informação é importante para pensar ações educativas tendo em vista o esclarecimento sobre os serviços de atendimento móvel, principalmente ações de cunho preventivo, direcionadas a discutir sobre o abuso de álcool e outras drogas.

O MS reconhece que a situação da saúde pública nacional, principalmente em nível de atenção às urgências, está marcada por uma série de falhas e, por essa razão, precisa ser reestruturada (Brasil, 2006). Segundo Rocha, Morais \& Benevides (2012), faz-se necessário que o poder público faça investimentos para melhoria dos serviços para que seja implementada e efetivada a Política Nacional de Redução de Morbimortalidade por Acidentes e Violências (Brasil, 2001). As autoras afirmam ainda que há necessidade de maior articulação entre os serviços de saúde pública, tendo em vista a complexidade das questões que envolvem tanto os acidentes quanto a violência de uma forma geral. Silva et al. (2009) estão de acordo com essa afirmativa, o que torna tal questão um potencial tema para ser mais discutido tendo em vista as possíveis 
melhorias para os serviços de atendimento móvel, bem como para a população e sociedade em geral, que se beneficiam desse serviço.

No artigo 2, Veronese, Oliveira e Nast (2012) tratam das Demandas não Pertinentes (DNPs), que, segundo as autoras, são definidas pelos médicos reguladores como situações que não conferem risco de vida. No contexto do estudo, foi observado que o perfil desse tipo de demanda se dá principalmente pela falta de orientação e conhecimento da população no que diz respeito à identificação de situações graves e ou não graves.

Os temas que envolvem a caracterização das DNPs são pontuados nos estudos como uma forma de encorajar as ações contínuas de gestão e planejamento de estratégias e dar subsídios a ela para que aperfeiçoem o atendimento e contribuam para o bom funcionamento dos serviços, reduzindo esse tipo de demanda.

Silva et al. (2009), no artigo 3, ao conhecerem a visão dos profissionais sobre o atendimento pré-hospitalar em Fortaleza, Brasil, identificaram questões referentes à gestão do Samu, que consequentemente interferem diretamente na assistência. O estudo expõe que a falta de infraestrutura, a escassez de recursos materiais e humanos, a falta de manutenção das ambulâncias e as falhas na integração com outros serviços são motivos de insatisfação dos profissionais, bem como sugere ainda a existência de deficiências e fragilidades na gestão que comprometem o desempenho e a qualidade da assistência à saúde oferecida.

Sendo assim, é necessário que os gestores do SUS contribuam para o fortalecimento do aperfeiçoamento da assistência oferecida pelo serviço de atendimento móvel, bem como que seja reforçada e implementada uma prática de avaliação no contexto desse serviço, tendo em vista melhoria e bom funcionamento do mesmo.

Algumas questões retratadas no artigo 4, escrito por Takeda e Robazzi (2007), descrevem situações e lacunas estruturais e organizacionais do Samu que contribuem para o aumento de chances de ocorrência de acidentes envolvendo condutores de ambulância. É destacado que o salário pago para motoristas de ambulância não é suficiente para seu sustento, por isso muitos trabalhadores possuem mais de um emprego, não tendo assim tempo apropriado para descanso. Além disso, o estudo aponta outros fatores, como o acúmulo de tarefas e desempenho de atividades que não lhes competem, por falta de diretrizes que respaldem as competências e responsabilidades; o prejuízo postural devido à ergonomia incorreta dos bancos dos automóveis; o desgaste físico imposto pelo peso relativo ao transporte de pacientes nas macas, entre outros (Takeda \& Robazzi, 2007).

Veronese, Oliveira e Nast (2012) tratam, no quinto artigo, de questões que envolvem a interpretação de risco de vida e de demandas não pertinentes e as implicações de tais fatos no contexto da enfermagem. As autoras reforçam que o trabalho da enfermagem no contexto gerencial das ações dos serviços de atendimento móvel se torna fundamental para a redução das DNPs, a organização do serviço, bem como a promoção de atividades educativas para esclarecer e educar a população sobre o serviço oferecido pelo Samu para que façam melhor uso dele.

A discussão sobre a percepção e o entendimento dos usuários sobre risco de vida revelou que este é um dos principais motivos que os levam a acionar tal serviço. Assim, a reflexão acerca da subjetividade do entendimento de risco de vida está diretamente relacionada com as demandas não pertinentes (Veronese; Oliveira \& Nast, 2012).

A Pnau determina a integração dos níveis assistenciais na atenção às urgências; a regulação médica; a capacitação pelos Núcleos de Ensino em Urgência (NEU); a regionalização; a gestão por comitês de urgência nos níveis municipal, regional, estadual e nacional; a humanização e a assistência centrada no usuário (Brasil, 2003). No entanto os temas das publicações analisadas neste estudo revelam lacunas no que tange à execução de tais determinações gerenciais propostas pela política.

As questões apontadas na literatura reforçam a necessidade de discussão entre gestores, profissionais e órgãos da saúde, para que sejam implementadas estratégias que possam colaborar para o funcionamento e a organização do Samu no 
âmbito do SUS, tendo em vista mais investimento no profissional e melhoria na comunicação e no sistema de referência e contrarreferência da rede de atenção a fim de que se efetive a integração dos serviços.

\subsection{Gestão dos Serviços de Atendimento Móvel: Capacitação profissional e Ações educativas para população}

Todos os estudos mencionam temas sobre a necessidade e a importância da capacitação profissional e do desenvolvimento de atividades educativas para a população tendo em vista o empedramento mediante a informação sobre identificação e reconhecimento de situações que envolvem risco de vida, funcionamento e objetivos dos Samus e principalmente sobre primeiros socorros.

A necessidade de capacitação e educação permanente para os profissionais que atuam nos serviços de atendimento móvel é um tema exposto em todos os cinco estudos selecionados que compuseram a amostra desta revisão. Silva et al. (2009) afirmam que, para qualificar os profissionais, faz-se necessário encaixar as atividades de educação permanente como carga horária trabalhada na rotina do serviço.

Ainda sobre a capacitação no contexto do Samu, Takeda e Robazzi (2007) reforçam a importância de que tal atividade seja oferecida para os motoristas ou condutores das ambulâncias, uma vez que a falta de conhecimento por parte desses profissionais pode aumentar também a probabilidade de risco de doenças ocupacionais.

Veronese, Oliveira \& Nast (2012) e Rocha, Morais \& Benevides (2012) reforçam que a educação permanente se faz necessária nesse contexto, bem como enfatizam o papel educador do enfermeiro, que, segundo os autores, deve desenvolver atividades educativas para a população sobre o serviço do Samu, primeiros socorros, entre outros temas, segundo a necessidade dos usuários. Veronese, Oliveira e Nast (2012) ressaltam que, na prática, ainda é evidenciado que muitos telefonemas recebidos pelo serviço visam buscar orientações médicas sobre primeiros socorros.

Silva et al. (2009) e Takeda e Robazzi (2007) destacam a necessidade de mais oferta e promoção da capacitação contínua para os profissionais que atuam no serviço de atendimento de saúde móvel. Assim, a capacitação, considerada também como um direito do trabalhador já destacado pela Pnau por meio dos Núcleos de Educação em Urgência (NEUs), deve possibilitar a reflexão sobre o trabalho tendo em vista a responsabilização e o comprometimento do profissional para com sua atuação (O’Dwyer \& Mattos, 2012).

Conforme O`Dwyer e Mattos (2012), a capacitação não se restringe somente a ofertar informações técnicas, contudo a deficiência em tais conhecimentos se torna uma barreira para efetivação das Pnaus. Assim, mesmo com a oferta de especialização em emergência para médicos e enfermeiros no Brasil, as publicações reforçam a necessidade de educação permanente, bem como enfatizam a importância de reconhecimento e valorização dos profissionais do Samu e de sua legitimidade junto aos serviços externos.

\section{Conclusão}

Sendo assim, diante da revisão apresentada, percebeu-se que existe uma diversidade de temas nas publicações científicas sobre os serviços de atendimento móvel de saúde, porém estes, de maneira (in)direta, relacionam-se com questões que estão interligadas à gestão de tal serviço. No entanto foi identificado que são poucas as publicações nacionais que versam sobre tal tema na biblioteca virtual de saúde, o que aponta a necessidade de realização de pesquisas que tenham como cenário o serviço de atendimento móvel de urgência enquanto cenário assistencial em saúde no contexto brasileiro, sendo está portanto, uma lacuna identificada na qual os autores recomendam a realização de novas investigações nos diferentes delineamentos metodológicos que sejam capazes de relatarem as peculiaridades envolvidas no cuidado de acordo com as diferentes demandas de urgência e de emergência no cotidiano do Samu. Destarte, este é um interesse de continuidade enquanto objeto de investigação da autora principal o qual será aprofundado em estudos futuros. 
Dessa forma, o conhecimento sobre os temas que estão sendo publicados no contexto da assistência na urgência e emergência é de extrema importância para o reconhecimento e a identificação de lacunas e/ou falhas na realização desse cuidado. O que consequentemente facilitará o desenvolvimento de propostas e estratégias para enfrentamento de problemas, visando alcançar melhorias na assistência.

Os principais temas encontrados foram organização do atendimento, demanda não pertinente, capacitação dos profissionais, promoção da educação para que a população conheça o que realmente é competência do Samu, entre outros aspectos que condizem com as perspectivas gerenciais, como estrutura material, recursos humanos e financiamento.

Assim, com base nas pesquisas descritas neste estudo, torna-se possível inferir que há necessidade de outras investigações que tenham como foco as demandas não pertinentes em outros contextos do serviço de atendimento móvel de urgência; protocolos e preenchimento de registros; tecnologias de gestão; serviços de referência e contrarreferência no contexto do Samu; reflexão da prática no cotidiano de trabalho; avaliação da Pnau. Vale salientar que são necessários estudos acerca de tais temáticas, a fim de subsidiarem a capacitação dos profissionais, bem como para fomentar discussão sobre o processo de trabalho no âmbito da gestão e das redes de atenção à saúde.

Esta revisão possibilitou observar que há uma necessidade latente de qualificação da gestão dos serviços de atendimento móvel, bem como da maior atuação dos comitês para que seja negociado o enfrentamento de barreiras operacionais, tendo em vista a facilitação de comunicação entre os serviços de urgências com os diferentes níveis de atenção à saúde (primária, secundária e terciária). Dessa maneira, além da atuação em rede, é necessário que profissionais sejam capacitados e se tornem atuantes politicamente na gestão do serviço, para que consigam melhores condições de trabalho para atender com qualidade e efetividade às demandas de saúde da população.

Os autores consideram uma limitação desta investigação, o recorte de tempo em que os artigos foram selecionados (indexados entre 2006 e 2016), porém, ressalta-se que ele foi condizente ao período em que a autora principal iniciava a construção do seu Trabalho de Conclusão de Curso (TCC) de Pós-graduação em Enfermagem em Urgência e Emergência justificando-se assim a não inclusão de investigações publicadas posteriormente na discussão dos resultados do presente artigo.

\section{Referências}

Brasil. (2006). Ministério da Saúde (MS). Política Nacional de Atenção às Urgências. (3a ed.), Brasília (DF): Editora do Ministério da Saúde.

Brasil. (2006). Ministério da Saúde (MS). Regulação Médica das Urgências. Brasília: MS.

Brasil. (2001). Ministério da Saúde (MS). Política Nacional de Redução da Morbimortalidade por Acidentes e Violências. Brasília: MS.

Gomes, P. S. D., \& Miranda, R. L. D. M. (2020). Atendimento pré-hospitalar e sua revalidação. E-book - bdex.eb.mil.br

Melo, L. D., Carvalho, A. W., Mello, J. L. B. V., Santos, P. R., Mozzer, D. D., Taroco, F. E. (2020). Assistência Intensiva a Pessoas com Insuficiência Cardíaca Descompensada na Prevenção e Tratamento do Edema Agudo Pulmonar. Revista Estação Científica. 2(24:1-20.

O’dwyer, G., \& Mattos, R. A. (2012). O SAMU, a regulação no Estado do Rio de Janeiro e a integralidade segundo gestores dos três níveis de governo. Physis Revista de Saúde Coletiva, 22(1): 141-60.

Rocha, E. G. A., Morais, A. C., \& Benevides, T. O. (2012). Serviço de atendimento móvel de urgência (SAMU) no município de Juazeiro-BA: principais especialidades demandadas. Revista Baiana de Saúde Pública, 36(4): 1041-1052.

Rosa, K. A., Oliveira, M. M., Ramos, C. I., Pereira, G. B., Alves, P. F., Rodriguez, M. L. B., \& Farias, T. A. (2020). Assistência do SAMU a pessoas sob efeito de substâncias psicoativas. Research, Society and Development, 9(11), e60791110024-e60791110024.

Silva, G. S., Mariot, M. D. M., \& Riegel, F. (2020). Profile of calls and drivers involved in motorcycle accidents by the mobile emergency service. Revista de Enfermagem da UFPI; 9:e9560.

Silva, J. G., Vieira, L. J. E. S., Pordeus, A. M. J., Souza, E. R., \& Gonçalves, M. L. C. (2009). Atendimento pré-hospitalar móvel em Fortaleza, Ceará: a visão dos profissionais envolvidos. Rev. Bras. Epidemiol, 12(4):591-603.

Sousa, L. M. M., Marques-Vieira, C. M. A., Severino, S. S. P., \& Antunes, A. V. (2017). A metodologia de revisão integrativa da literatura em enfermagem. Revista Investigação em Enfermagem, 21(2):17-26. 
Research, Society and Development, v. 10, n. 2, e38310212522, 2021

(CC BY 4.0) | ISSN 2525-3409 | DOI: http://dx.doi.org/10.33448/rsd-v10i2.12522

Takeda, E., \& Robazzi, M. L. C. C. (2007). Acidentes de trabalho com motoristas de ambulâncias que realizam socorro de urgência. Rev. Latino-Am. Enfermagem, 15(3): 439-445.

Veronese, A. M., Oliveira, D. L. L. C., \& Nast, K. (2012). Risco de vida e natureza do SAMU: demanda não pertinente e implicações para a enfermagem. Rev. Gaúcha Enferm, 33(4): 142-148.

Zerbetto Filho, J. A. A., de Souza, M. P., \& Carvalho, M. D. B. (2020). Perfil epidemiológico dos atendimentos psiquiátricos pelo SAMU Norte Novo no ano de 2018. Research, Society and Development, 9(10), e7459109122-e7459109122. 